\title{
Subband Structure of II-VI Modulation-Doped Magnetic Quantum Wells
}

\author{
Henrique J. P. Freire* and J. Carlos Egues ${ }^{\dagger}$ \\ Departamento de Física e Informática, Instituto de Fúsica de São Carlos, \\ Universidade de São Paulo, 13560-970 São Carlos, São Paulo, Brazil
}

Received on 23 April, 2001

\begin{abstract}
Here we investigate the spin-dependent subband structure of newly-developed Mn-based modulation-doped quantum wells. In the presence of an external magnetic field, the $s$ - $d$ exchange coupling between carriers and localized $d$ electrons of the Mn impurities gives rise to large spin splittings resulting in a magnetic-field dependent subband structure. Within the framework of the effective-mass approximation, we self-consistently calculate the subband structure at zero temperature using Density Functional Theory (DFT) with a Local Spin Density Approximation (LSDA). We present results for the magnetic-field dependence of the subband structure of shallow $\mathrm{ZnSe} / \mathrm{ZnCdMnSe}$ modulation doped quantum wells. Our results show a significant contribution to the self-consistent potential due to the exchange-correlation term. These calculations are the first step in the study of a variety of interesting spin-dependent phenomena, e.g., spin-resolved transport and many-body effects in polarized two-dimensional electron gases.
\end{abstract}

Recent advances in nanoengineering of Mn-based semiconductor heterostructures open up an exciting area of research which combines both magnetism and quantum size effects. The successful achievement of high-doping carrier densities in Mn-based II-VI layered compounds [1] provides a new and promising ground for research: spin-polarized two-dimensional magnetotransport in (semi)magnetic wells. Recently, Smorchkova et al. [2] reported Hall-effect measurements in spin-polarized two-dimensional electron gases in novel $n$-doped digital-magnetic heterostructures $[3,4]$.

Here we investigate the magnetic-field dependence of the spin-dependent electronic structure of a modulation-doped digital magnetic quantum well (QW) [2,5-7] using Density Functional Theory (DFT) with $a$ Local Spin Density Approximation (LSDA). We consider a Digital Magnetic Heterostructure (DMH) [5] in which magnetic Mn atoms are digitally incorporated in a planar configuration inside the well. In the presence of an external magnetic field, the $s$ - $d$ exchange coupling between carriers and localized $d$ electrons of the Mn impurities yields large spin splittings that exceed the inter-Landau level (LL) spacing $\hbar \omega_{c}$ [8]. This gives rise to a magnetic-field dependent subband structure and a net spin-polarization of the electron gas even at low magnetic fields.

\footnotetext{
${ }^{*}$ Electronic address: freire@if.sc.usp.br

$\dagger$ Electronic address: egues@if.sc.usp.br
}

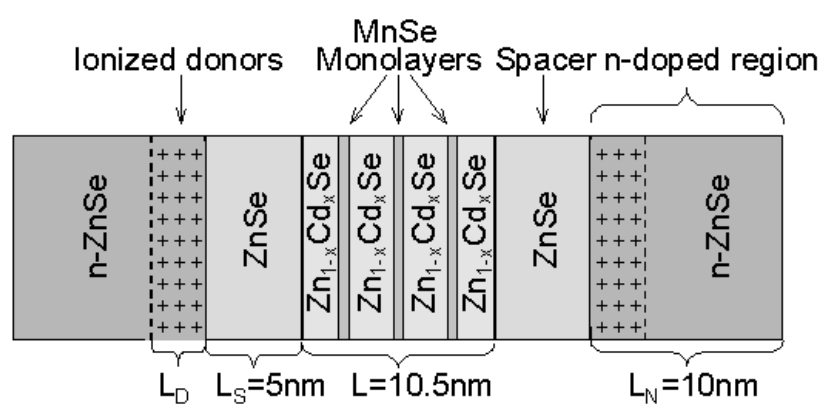

Figure 1. Modulation-doped semimagnetic quantum well: the structure consists of ZnSe layers sandwiching a ZnCdSe layer containing digitally-incorporated MnSe monolayers. Adjacent to the $\mathrm{ZnSe}$ spacer there are $n$-doped regions (electron reservoirs). Two depleted regions of length $L_{D}$ arise within the $n$-doped layer as the doping electrons move into the well.

Physical system. We consider the modulationdoped semi-magnetic quantum well shown in Fig. 1. The QW structure consists of ZnSe layers surrounding a 35-monolayer (ML) ZnCdSe/MnSe heterostructure with six equispaced digitally-incorporated $\mathrm{MnSe}$ monolayers, denoted by $6 \times \frac{1}{2}$ ML after Crooker et al. [9]. This notation means there are $6 \mathrm{Mn}$ planes with nominal planar concentration $x_{p}=1 / 2$. Adjacent to each ZnSe spacer, there are $n$-doped regions (impurity concentration $N_{i}=1.2 \times 10^{17} \mathrm{~cm}^{-3}$ ) that serve as electron reservoirs. Electrons from the $n$-doped regions fill 
up the confined energy levels of the QW thus forming a two-dimensional electron gas (2DEG) and leaving behind depleted regions of length $L_{D}$ in the reservoirs. As the magnetic field is changed, we expect modifications in the self-consistent potential and in the corresponding bandstructure due to $i$ ) magnetic-field dependent contributions to the confining potential and $i i)$ rearrangement of electrons in the confined levels because of the magnetic-field dependent Landau-level degeneracy $e B / h$.

DFT/LSDA approach. Within Density Functional Theory, we solve the self-consistent Kohn-Sham equations using $a$ Local Spin Density Approximation scheme to obtain the magnetic field dependent subband structure, modulation-doped confining potential, and carrier concentration. We consider the system at zero temperature and perform the calculations within the framework of the effective-mass approximation. The dominant contribution to the magnetization comes from the localized moments of the Mn ions. These are treated as a paramagnetic collection of ions by using an effective Brillouin function. However, we also consider the effects of the spin density on the exchange-correlation (XC) term. This is done within the interpolation scheme of Barth and Hedin [10] as parametrized by Gunnarsson and Lundqvist [11]. Since the energy scale in our system (tens of $\mathrm{meV}$ ) is the same as that of the calculated $\mathrm{XC}$ contribution to the confining potential (up to $18 \mathrm{meV}$ ), we expect XC to significantly affect the self-consistent subband structure. Thus, to assess the role of exchange and correlation in the electronic structure in these novel geometries, we contrast our DFT/LSDA results with similar ones within the Hartree approximation [12].

Results and discussions. We present results for shallow ZnSe/ZnCdMnSe modulation-doped QWs. By shallow we mean the confining potential $(25 \mathrm{meV})$ is small enough so that $(\sim) 10.5 \mathrm{~nm}$ wide wells have only a few confined subbands. For these geometries, $n$-doped regions with concentrations near $10^{17} \mathrm{~cm}^{-3}$ provide carriers that fill up all the QW levels thus serving as a reservoir of electrons ("reservoir hypothesis" [13]). We obtain the magnetic-field dependence of the subband structure and our results show a significant contribution to the self-consistent potential due to the XC term. We have calculated the self-consistent potential profile, electronic structure and the two-dimensional electronic density for magnetic fields ranging from zero to ten tesla.

Fig. 2 shows the potential profile, subband energy and wavefunction squared for spin-down electrons at $B=1 \mathrm{~T}$. The potential profile consists of the structural confining potential, the magnetic field and spindependent $s$ - $d$ exchange energy within the magnetic monolayers, the Hartree contribution, the Zeeman energy, and the spin-dependent XC energy. The resulting self-consistent potential has the usual modulationdoped profile that arises from the addition of the
Hartree energy of the confined electrons to the confining potential. We contrast the LSDA calculation to the Hartree one. First we note that, despite the self-consistent Hartree result being fully converged, the LSDA energy profile is not flat at both ends as one would expect. The reason for this behavior may be that the LSDA parametrization of Gunnarsson and Lundqvist [11] is not appropriate to describe $\mathrm{XC}$ at very low electronic densities such as those within the wavefunction tails [their results are for Wigner-Seitz radius $r_{s}$ (in units of effective Bohr radius) within the range $1-9$, while our data has $r_{s}$ from 20 to 300 at the tails]. Nevertheless, as most of the wave function is confined inside the well region, we do not expect these non-flat regions to drastically affect the electronic structure of the system. In fact, we have tested this hypothesis by abruptly setting the XC contribution to zero for $r_{s}$ greater than 9 and we have not found any substantial deviation in our results.

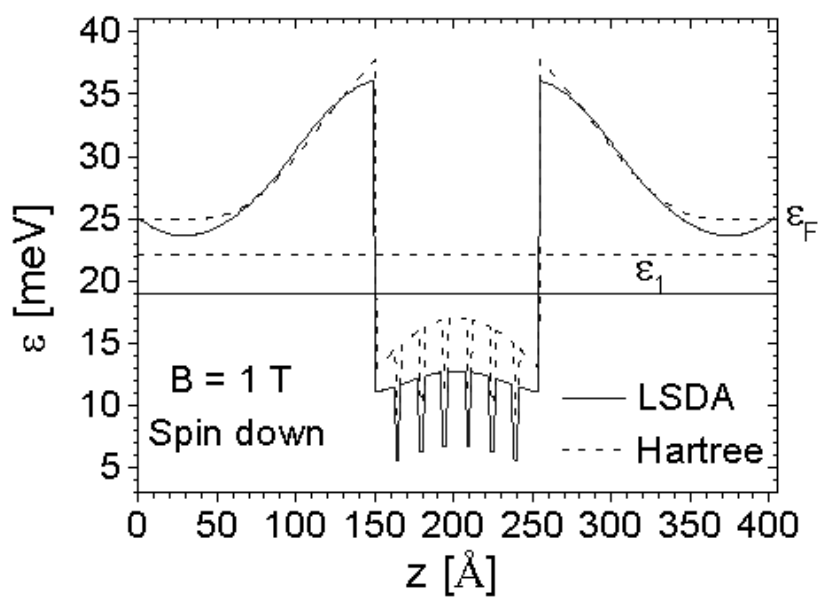

Figure 2. Calculated modulation-doped potential profile, subband energy $\varepsilon_{1}$ and wavefunction squared for spin-down electrons in the structure shown in Fig. 1 within the Hartree (dashed lines) and the LSDA (solid lines) approximations. The Fermi energy $\varepsilon_{F}$ is set by the chemical potential of the reservoir, arbitrarily set to $25 \mathrm{meV}$. The main effect of the XC contribution in the LSDA calculation is to lower the confining potential and the subband energy $\varepsilon_{1}$ for the spin-down electrons when compared to the Hartree case.

We can clearly see from Fig. 2 that the XC contribution significantly lowers the energy $\varepsilon_{1}$ for the spin-down electrons. This effect is larger for the spin-down electrons than for spin-up electrons because the electron gas is spin-down polarized. Pauli's exclusion principle allows (does not allow) a spin up (down) electron and the majority spin down electrons to overlap thus raising (lowering) the electrostatic energy.

In Fig. 3 we plot the first three spin-resolved Landau-level fan diagrams for both the Hartree (a) and the LSDA (b) cases. Note that the XC enhances the spin splitting because it decreases (increases) the spin down (up) energy in the case of a spin-down polarized gas. The second point is that the LLs are pinned to the 
Fermi level $\varepsilon_{F}$ for distinct ranges of magnetic fields (see plateaus at $\varepsilon_{F}$ ) [14]. In the present model, this follows from the "reservoir hypothesis" which allows for two competing mechanisms to act: $i$ ) as the magnetic field increases, the LLs energies also increase and cross the Fermi energy, thus expelling electrons out of the quantum well; ii) on the other hand, less electrons means less Coulomb repulsion and the energy tends to lower. An equilibrium between these two mechanisms is responsible for the plateaus. Note that the pinning of the Landau level in the present model is different from the standard one used to describe the Integer Quantum Hall Effect (IQHE) [15]. There, localized states in broadened Landau levels are responsible for the Fermilevel pinning [16].

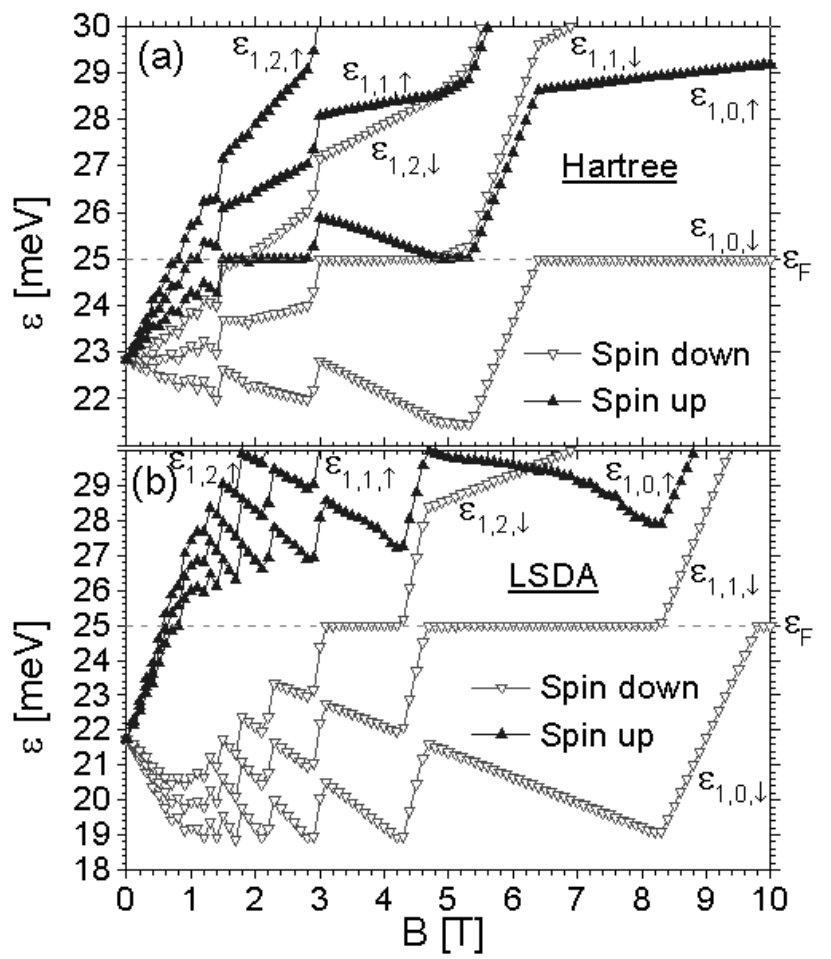

Figure 3. Fan diagrams for the first three spin-down (triangle down) and spin-up (triangle up) Landau levels for both Hartree (a) and LSDA (b) calculations. Note that the exchange-correlation contribution enhances the spinsplitting (b) and makes the 2DEG fully polarized at lower magnetic field $(\sim 1 \mathrm{~T})$ than in the Hartree case $(\sim 3 \mathrm{~T})$. The Fermi level $\varepsilon_{f}$ (dashed line) remains constant at $25 \mathrm{meV}$ (electron reservoir) while the Landau levels oscillate as the spin-dependent potential profile changes with the magnetic field.

One experimental relevant quantity affected by the electronic structure is the two-dimensional electron density $n_{2 D}$. At zero magnetic field we find that $n_{2 D}$ is $2.175 \times 10^{11} \mathrm{~cm}^{-2}$ and $1.444 \times 10^{11} \mathrm{~cm}^{-2}$, respectively, for LSDA and Hartree calculations. This considerable difference comes about because $\mathrm{XC}$ lowers the subband energies as compared to the Hartree case thus leading to an effective increase in $n_{2 D}$.
We have studied the self-consistent electronic structure of a modulation-doped semimagnetic quantum well including exchange and correlation of electrons within the DFT/LSDA scheme. We found that for shallow geometries the exchange-correlation contribution is significant to the electronic structure as it $i$ ) lowers the subband energy of the majority spin-down electrons and ii) enhances the spin-splitting thus making the electron gas fully spin-polarized at lower magnetic fields $(\sim 1 \mathrm{~T})$ than in the Hartree case $(\sim 3 \mathrm{~T})$. Our results are preliminary but do point to the relevance of the $\mathrm{XC}$ in shallow ZnSe semimagnetic QWs, in contrast to GaAs systems. The calculations reported here are a first step in the study of a variety of interesting spin-dependent phenomena, e.g., spin-resolved transport and many-body effects in spin-polarized two-dimensional electron gases.

This work is being supported by FAPESP (99/06868-3), Brazil. We thank Prof. Nitin Samarth (PennState) for suggesting this problem to us. One of us (HJPF) acknowledges the hospitality at Prof. Samarth's group during his visit.

\section{References}

[1] I. Smorchkova and N. Samarth, Appl. Phys. Lett. 69, 1640 (1996).

[2] I. P. Smorchkova, N. Samarth, J. M. Kikkawa, and D. D. Awschalon, Phys. Rev. Lett. 78, 3571 (1997).

[3] Longitudinal magnetotransport in Mn-based heterostructures has also been recently achieved. See, for instance, R. Fiederling et al., Nature (London) 402, 787 (1999); Y. Ohno et al., Nature (London) 402, 790 (1999); and B. T. Jonker et al., Phys. Rev. B 62, 8180 (2000). See also G. Schmidt et al., Phys. Rev. Lett. 87, 227203 (2001).

[4] For theoretical works on longitudinal spin-polarized transport see J. C. Egues, Phys. Rev. Lett. 80, 4578 (1998); J. C. Egues, C. Gould, G. Richter, and L. W. Molenkamp, Phys. Rev. B 64, 195319 (2001); K. Chang and F. M. Peeters, Solid State Commun. 120, 181 (2001); and Y. Guo, J. Lu, B. Gu, and Y. Kawazoe, Phys. Rev. B 64, 155312 (2001).

[5] D. D. Awschalom and N. Samarth, J. Magn. Magn. Mater. 200,130 (1999).

[6] R. Knobel, N. Samarth, S. A. Crooker, and D. D. Awschalom, Physica E 6, 786(2000).

[7] J. J. Berry, R. Knobel, O. Ray, W. Peoples, and N. Samarth, J. Vac. Sci. Technol. B 18, 1692 (2000).

[8] J. K. Furdyna, J. Appl. Phys. 64, R29 (1988).

[9] S. A. Crooker et al., Phys. Rev. Lett. 75, 505 (1995).

[10] V. von Barth and L. Hedin, J. Phys. C 5, 1629 (1972).

[11] O. Gunnarsson and B. I. Lundqvist, Phys. Rev. B 13, 4274 (1976).

[12] The DFT/LSDA scheme accounts for XC. The Hartree approximation is obtained by setting $\mathrm{XC}$ to zero in this scheme. 
[13] G. A. Baraff and D. C. Tsui, Phys. Rev. B 24, 2274 (1981); A. Raymond and S. Sibari. Phys. Stat. Sol. b 183, 159 (1994)

[14] $\varepsilon_{F}$ remains constant at the chemical potential of the reservoir. Note that because the quantum well is shallow and the donor concentration within the $n$-doped regions is high, this region is never completely depleted.

[15] K. von Klitzing, G. Dorda, and M. Pepper, Phys. Rev. Lett. 45, 494 (1980).

[16] H. L. Stormer and D. C. Tsui, Science 220, 1241 (1983). 